\title{
Development of a new analytical spectroscopic methodology based on the competitive aggregation in a dye-surfactant-drug system: Application to the determination of gemfibrozil
}

\author{
Mohammad Amjadi *, Jamshid L. Manzoori and Leila Farzampour \\ Department of Analytical Chemistry, Faculty of Chemistry, University of Tabriz, Tabriz, Iran
}

\begin{abstract}
A new analytical methodology based on the competitive aggregation in a dye-surfactant-drug system is developed for the determination of gemfibrozil. Eriochrome Blue Black R (EBBR) and Didodecyldimethylammonium bromide (DDABr) were the dye and surfactant used, respectively. In the proposed method, the anions of the dye bind to the cationic surfactant molecules to form dye-surfactant aggregates, which are monitored from changes in UV-Vis absorption features of the dye. In the ternary EBBR-DDABr-drug mixtures, the drug competes with the dye to interact with the surfactant, which results in a decrease in dye-surfactant aggregates formation. This, again, causes a change in absorption properties of the dye. The measurement parameter is the difference between the absorption of the dye in the presence and absence of the drug. In the appropriate experimental conditions the absorbance differences are directly proportional to the drug concentration. The influence of several experimental variables such as $\mathrm{pH}$, concentrations of buffer, EBBR and DDABr on the measurement parameter were studied. Under the optimum conditions, the calibration graph was linear up to $6.0 \mu \mathrm{g} \mathrm{ml}{ }^{-1}$ with the correlation coefficient of 0.998 . The limit of detection and quantification were found to be 0.044 and $0.15 \mu \mathrm{g} \mathrm{ml}{ }^{-1}$, respectively. The method was validated and applied to the determination of gemfibrozil in pharmaceutical preparations.
\end{abstract}

Keywords: Surfactant, mixed aggregate, dye-surfactant aggregate, gemfibrozil

\section{Introduction}

Surfactants are amphiphilic molecules that have both hydrophobic and hydrophilic groups. As a result of this special structure, surfactants can form several kinds of self-assembly associations in bulk solution including normal and reverse micelles, bilayers, vesicles and etc. It is well known that these assemblies can alter some physicochemical properties of various solutes. Based on this capability, surfactant solutions have been used in many analytical procedures to improve their performances [1,2].

Recently, Pérez-Bendito and coworkers have developed two analytical methodologies based on the capability of surfactants to form mixed aggregates with other molecules of appropriate properties. In

\footnotetext{
*Corresponding author. E-mail: amjadi@tabrizu.ac.ir.
} 
one of these methods (named as mixed aggregate method), critical micelle concentration (CMC) of a surfactant is used as a measurement parameter. In the presence of an amphiphilic analyte the mixed analyte-surfactant aggregates is formed, which results in substantially lower CMCs than would be expected for surfactant alone [3,4]. The second method (named as the surfactant to dye binding degree method) is based on the effect of amphiphilic compound on the degree of binding of surfactant to dye molecules [5-8]. In the method, a cationic surfactant such as Didodecyldimethylammonium bromide (DDABr) and an anionic dye, usually Commassie Brilliant Blue $\mathrm{G}$ (CBBG) has been used. The anions of dyes bind to cationic surfactant molecules to form dye-surfactant aggregates, which are monitored from changes in UV-Vis absorption features of the dye. In the ternary CBBG-DDABr-analyte mixtures, the analyte competes with the dye to interact with the surfactant, which results in a decrease in the degree of binding of surfactant to dye molecules proportional to the analyte concentration. For each standard or sample a photometric titration by surfactant solution is needed to obtain the corresponding measurement parameter. This makes the method very time consuming though it is versatile and has low cost.

Gemfibrozil, 5-(2,5-dimethylphenoxy)-2,2-dimethylpentanoic acid (Fig. 1(a)), is a fibrate hypolipidemic agent that is clinically effective in reducing serum cholesterol and triglyceride levels. It has also been demonstrated that this drug lowers the incidence of coronary heart disease in humans $[9,10]$. Several reports on gemfibrozil determination have appeared in the literature. Gas chromatography [11] and high performance liquid chromatography (HPLC) with ultraviolet or fluorimetric detection [12-19] have been used for the determination of gemfibrozil and its metabolites in plasma and urine samples. A liquid chromatography-mass spectrometric method [20] has been reported for the determination of this drug in water and wastewater samples. Near-infrared diffuse reflectance spectroscopy [21-24] has been applied to the determination of gemfibrozil in pharmaceutical preparations. Spectrofluorimetric and micelleenhanced spectrofluorimetric methods [25] and flow injection spectrofluorimetry [26] have also been reported for its determination in pharmaceutical preparations. The only spectrophotometric method proposed for the determination of gemfibrozil is based on the direct measurement of its absorbance in basic medium [27].

In the present work, we have modified the surfactant to dye binding degree methodology to make it simpler and less time consuming. Eriochrome Blue Black R (EBBR) and DDABr were the dye and surfactant used, respectively. The measurement parameter is the difference between the absorption of the dye in the presence and absence of the drug. In the certain chemical conditions, the absorbance

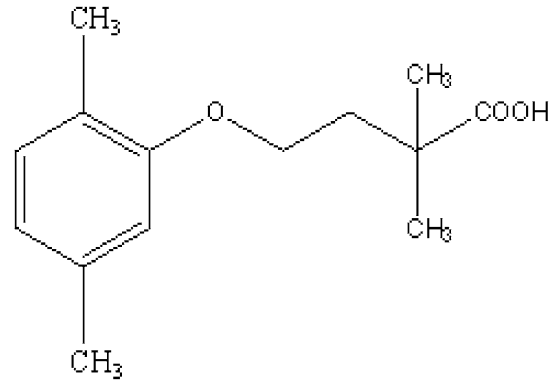

(a)

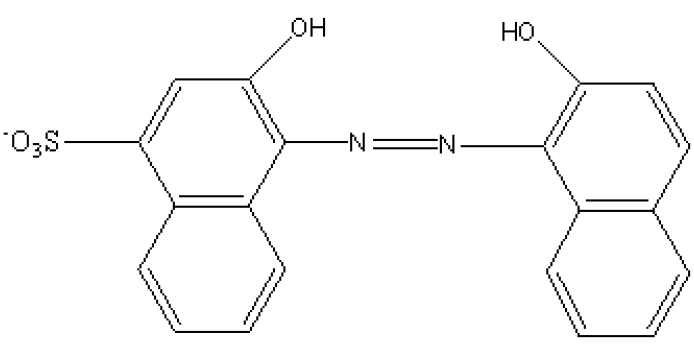

(b)

Fig. 1. Structures of (a) gemfibrozil and (b) EBBR. 
differences were found to be directly proportional to the drug concentration. The proposed method was used for the determination of gemfibrozil as a model drug.

\section{Experimental}

\subsection{Apparatus}

Absorption spectra were recorded by Cary model 100 spectrophotometer (Varian) and absorbance measurements were made on a Shimadzu UV-120-02 spectrophotometer. $1.0 \mathrm{~cm}$ quartz cells were used. A Metrohm model $654 \mathrm{pH}$ meter was used for $\mathrm{pH}$ measurements.

\subsection{Reagents}

All reagents used were of analytical reagent grade. Doubly distilled water was used throughout.

Gemfibrozil (Dipharma, Italy) was kindly provided with Shahre Darou Laboratory. A stock standard solution of $1000 \mu \mathrm{g} \mathrm{ml}^{-1}$ was prepared by dissolving $0.10 \mathrm{~g}$ of gemfibrozil in approximately $10 \mathrm{ml}$ $0.1 \mathrm{M} \mathrm{NaOH}$ solution and diluting to mark in $100 \mathrm{ml}$ volumetric flask with water. A $2.5 \times 10^{-4} \mathrm{M}$ solution of Eriochrome Blue Black R (EBBR, Merck) and a $5 \times 10^{-4}$ M solution of Didodecyldimethylammonium bromide (DDABr, Acros Chemical Company) were prepared in distilled water.

\subsection{Recommended procedure}

To a set of $25 \mathrm{ml}$ volumetric flasks containing various amounts of gemfibrozil, $1 \mathrm{ml} 0.25 \mathrm{M}$ acetate buffer, $3.25 \mathrm{ml} 2.5 \times 10^{-4} \mathrm{M}$ EBBR and $2.25 \mathrm{ml} 5 \times 10^{-4} \mathrm{M}$ DDABr were added and diluted to volume by water. The final concentration of gemfibrozil in these solutions should be in the range of $0.2-6.0 \mu \mathrm{g} \mathrm{ml}^{-1}$. The solutions were stirred for about $5 \mathrm{~min}$ by using small magnets. Then the absorbance values were measured at $661 \mathrm{~nm}$ against the reagent blank. A similar solution was also prepared without adding drug and its absorbance was measured at the same wavelength and against the same blank. The absorbance differences between the standard solutions and the latter solution $\left(\Delta A=A_{s}-A_{r}\right)$ were calculated and plotted vs. the drug concentration.

\subsection{Pharmaceutical preparation}

Ten capsules of gemfibrozil were weighed in order to find the average mass of each capsule. Then the contents were powdered and mixed. A portion of $10.0 \mathrm{mg}$ of this powder was accurately weighed and dissolved in about $10 \mathrm{ml} 0.1 \mathrm{M} \mathrm{NaOH}$ solution and filtered into a $100 \mathrm{ml}$ volumetric flask. The residue was washed several times with water and the solution was diluted to the mark with water. A suitable aliquot of this solution was taken for the spectrophotometric measurement of gemfibrozil according to the recommended procedure. The recovery assay was carried out using the same procedure but adding the known amounts of gemfibrozil.

\section{Results and discussion}

\subsection{Study of the dye-surfactant interaction}

It is well known that the interaction of a given surfactant at concentrations less than CMC, with dye molecules of having opposite charges leads to the formation of mixed dye-surfactant premicellar 
aggregates $[28,29]$. This may changes the spectral properties of the dye as well as its acidity constants $[2,28]$. EBBR $\left(\mathrm{H}_{2} \mathrm{D}^{-}\right.$, Fig. $\left.1(\mathrm{~b})\right)$ has two ionizable protons and the first $\mathrm{p} K_{a}$ value $\left(\mathrm{H}_{2} \mathrm{D}^{-}=\mathrm{HD}^{2-}+\right.$ $\mathrm{H}^{+}$) was found to be 6.3 in our experimental conditions. Upon addition of $4.5 \times 10^{-4} \mathrm{M}$ DDABr, this acidity constant is shifted to 4.8 . This can be attributed to the fact that cationic surfactant $\operatorname{DDABr}\left(\mathrm{S}^{+}\right)$ in the presence of anionic dye EBBR can forms mixed EBBR-DDABr aggregates. Because of greater electrostatic attraction between $\mathrm{HD}^{2-}$ anions and DDABr cations, these anions are more tightly bound to the surfactant molecules than $\mathrm{H}_{2} \mathrm{D}^{-}$anions shifting the dissociation equilibrium to the right (decreasing $\mathrm{p} K_{a}$ value). The relationship between two $\mathrm{p} K_{a}$ values can be written as follows [30]:

$$
\mathrm{p} K_{a}^{\prime}-\mathrm{p} K_{a}=-\log \left(1+\beta_{d}\left[\mathrm{~S}^{+}\right]^{m / n}\right),
$$

where $\mathrm{p} K_{a}^{\prime}$ is acidity constant in the presence of surfactant (conditional acidity constant), $\beta_{d}$ is the dyesurfactant binding constant, $\left[\mathrm{S}^{+}\right]$is the free surfactant concentration and $m / n$ is the ratio of stoichiometric coefficients of surfactant to dye in mixed aggregates (see the following scheme). The formation of dye-surfactant aggregates can be shown as the following scheme:

$$
m \mathrm{~S}^{+}+n \mathrm{HD}^{2-} \longrightarrow(\mathrm{HD})_{n} \mathrm{~S}_{m}^{(2 n-m)-} .
$$

The formation of aggregates can also be confirmed by monitoring changes in spectral features of EBBR. Figure 2 shows the absorption spectra of EBBR in the presence of various concentrations of DDABr. As can be seen the spectral features of the dye vary as a function of surfactant concentration. This is due the fact that EBBR-DDABr binding degree is dependent on the DDABr/EBBR molar ratio. Figure 3 shows the plot of absorbance of the dye at $661 \mathrm{~nm}$ as a function of DDABr concentration. The broken line obtained indicates the formation of EBBR-DDABr aggregates of two different well-defined stoichiometries in proportion to DDABr concentration (i.e. $m / n: 3 / 4$ and $3 / 2$ ).

\subsection{Interaction of gemfibrozil with EBBR-DDABr aggregates}

The addition of gemfibrozil to EBBR-DDABr system (at pH 5.5 and DDABr/EBBR molar ratio higher than 0.5 ) leads to the modification of the spectral features of the dye (Fig. 2). This effect can be contributed to the competition of the gemfibrozil with the dye to form gemfibrozil-DDABr mixed aggregates. The interaction can be confirmed by study of UV absorption properties of gemfibrozil in various DDABr concentrations (Fig. 4). As can be seen from Fig. 4A the addition of DDABr to gemfibrozil solution results in an increase in the absorbance measured for the drug in UV region. This can be explained on the basis of electrostatic interaction between anions of gemfibrozil $\left(\mathrm{G}^{-}\right)$and cations of $\operatorname{DDABr}\left(\mathrm{S}^{+}\right)$ to form gemfibrozil-DDABr mixed aggregates as follows:

$$
\begin{aligned}
& \mathrm{G}^{-}+\mathrm{S}^{+} \longrightarrow \mathrm{GS}, \\
& \mathrm{GS}+\mathrm{S}^{+} \longrightarrow \mathrm{GS}_{2}^{+}, \\
& \mathrm{GS}_{p-1}^{(p-2)+}+\mathrm{S}^{+} \longrightarrow \mathrm{GS}_{p}{ }^{(p-1)+} .
\end{aligned}
$$

Figure 4B shows the results obtained by plotting the variation of absorbance of gemfibrozil at $275 \mathrm{~nm}$ as a function of DDABr/gemfibrozil molar ratio. The broken line obtained indicates the formation of DDABr:gemfibrozil aggregates of different stoichiometries (between 1:1 and 4:1) as DDABr concentration increases. From these studies it can be assumed that DDABr forms premicellar aggregates with 


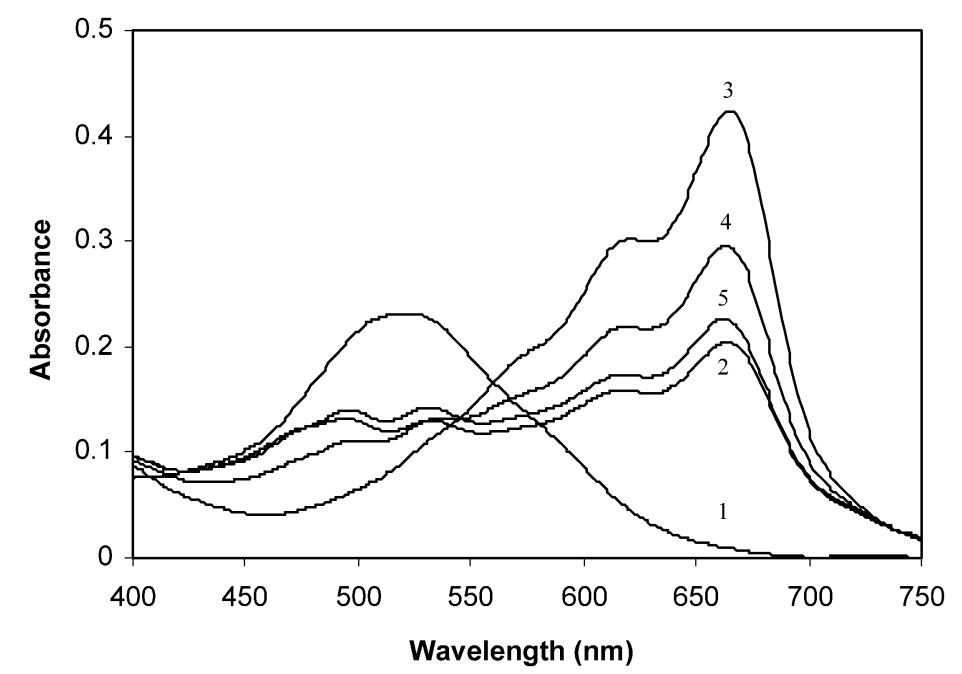

Fig. 2. Absorption spectra of $\operatorname{EBBR}\left(3.25 \times 10^{-5} \mathrm{M}\right)$ at $\mathrm{pH} 5.5$, (1) in the absence of DDABr, $(2,3)$ in the presence of (2) $3 \times 10^{-5}$ and (3) $4.5 \times 10^{-5} \mathrm{M} \mathrm{DDABr}$, (4, 5) same as (4) but in the presence of (4) 2.4 and (5) $4.0 \mu \mathrm{g} \mathrm{ml}^{-1}$ gemfibrozil.

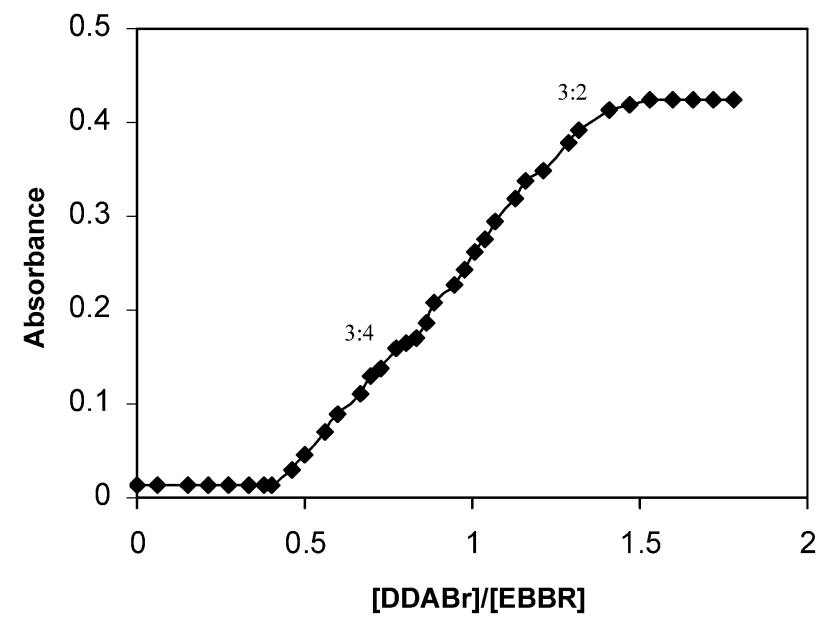

Fig. 3. Variation of the absorbance of $\operatorname{EBBR}\left(3.25 \times 10^{-5} \mathrm{M}\right)$ at $661 \mathrm{~nm}$ as a function of DDABr/EBBR molar ratio at $\mathrm{pH} 5.5$.

both EBBR and gemfibrozil molecules, and that the addition of gemfibrozil to the EBBR-DDABr system strongly affects the extents of EBBR-DDABr mixed aggregate formation. This phenomenon can be monitored from the changes in absorbance of EBBR at a suitable wavelength. This forms the basis of the analytical methodology for the determination of gemfibrozil.

\subsection{Measurement parameter}

In the methodology developed by Pérez-Bendito and coworkers, the concentration of analyte (drug) is determined from the following equation:

$$
m_{S}^{*}-m_{S}=\beta_{A} m_{A},
$$




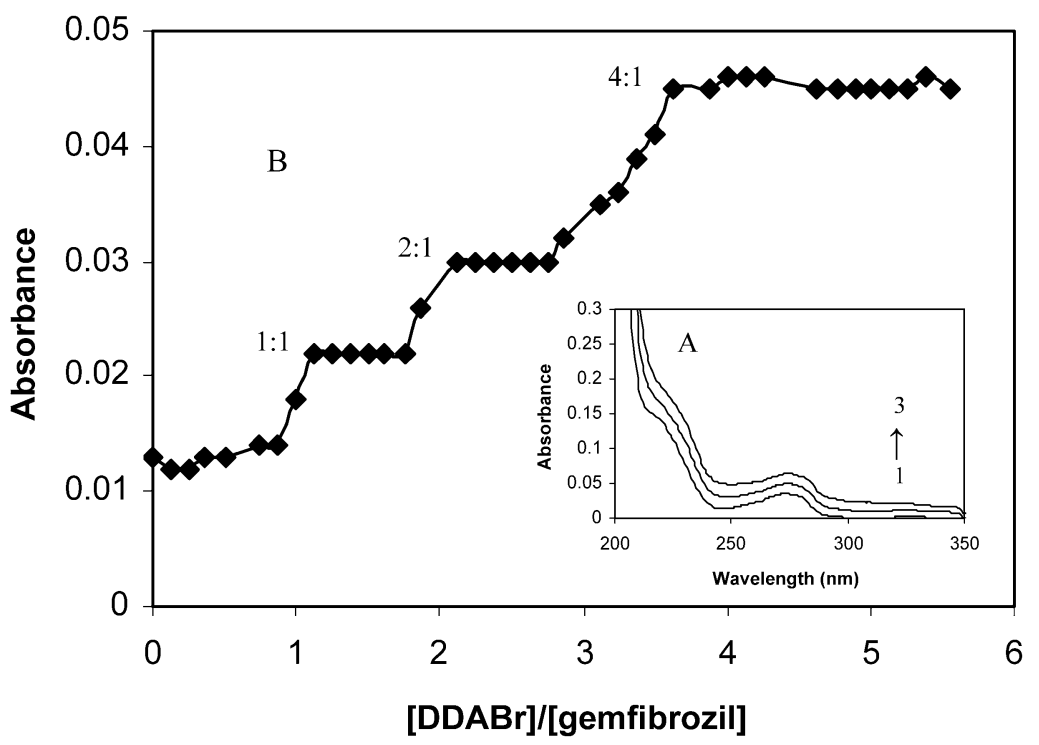

Fig. 4. (A) Absorption spectra of gemfibrozil $(16 \mu \mathrm{M})$ in (1) the absence of surfactant and (2,3) the presence of (2) $2 \times 10^{-5} \mathrm{M}$ and (3) $4 \times 10^{-5} \mathrm{M} \mathrm{DDABr}$ at $\mathrm{pH}$ 5.5. (B) Variation of the absorbance of gemfibrozil $(16 \mu \mathrm{M})$ at $275 \mathrm{~nm}$ as a function of $\mathrm{DDABr} / \mathrm{gemfibrozil} \mathrm{molar}$ ratio at $\mathrm{pH}$ 5.5.

where $m_{S}^{*}$ and $m_{S}$ denote the surfactant concentration required to reach a given degree of dye-surfactant binding in the presence and absence of the drug, respectively, $m_{A}$ the concentration of the drug and $\beta_{A}$ surfactant-drug binding degree. Calibration graph is constructed by plotting $m_{S}^{*}-m_{S}$ as a function of the drug concentration. For the calculation of $m_{S}^{*}$ and $m_{S}$ the photometric titration by surfactant solution should be performed in the presence and absence of drug. Obviously, these steps render the methodology very time consuming and tedious. However, our preliminary works showed that in the appropriate $\mathrm{pH}$ and DDABr/EBBR molar ratio (i.e. the conditions in which $\left[\mathrm{HD}^{2-}\right] /\left[\mathrm{H}_{2} \mathrm{D}^{-}\right]$ratio and therefore solution absorbance can dramatically change by adding drug), the differences between absorbance of EBBRDDABr solution $\left(A_{r}\right)$ and EBBR-DDABr-drug solutions $\left(A_{s}\right)$ are directly proportional to the drug concentration. Therefore, in order to obtain a linear calibration graph, we simply plot the absorbance differences $\left(\Delta A=A_{s}-A_{r}\right)$ as a function of drug concentration. This makes the method very simple and rapid.

\subsection{Optimization of variables}

The effect of several experimental variables on the chemical system have been investigated and optimized to obtain maximum sensitivity.

The influence of $\mathrm{pH}$ on the measurement parameter $(\Delta A)$ was studied in the range of 4-7. The results are shown in Fig. 5(a). As can be seen the absorbance difference is reached a maximum at $\mathrm{pH}$ 5.5. Therefore this $\mathrm{pH}$ was chosen as optimum value. At $\mathrm{pH}$ values lower than 4 , gemfibrozil $\left(\mathrm{p} K_{a}=4.7\right)$ is present in the protonated form and so is not able to form mixed aggregates with surfactant molecules. When the $\mathrm{pH}$ of solution is higher than about 7 , the ratio $\left[\mathrm{HD}^{2-}\right] /\left[\mathrm{H}_{2} \mathrm{D}^{-}\right]$is so great that solution absorbance would not be changed by adding the drug. Acetate buffer was used for the adjustment of $\mathrm{pH}$ and the effect of its concentration was studied. According to the results, increasing the buffer concentration decreases the measurement parameter. Electrolytes are known to decrease the electrostatic interactions 


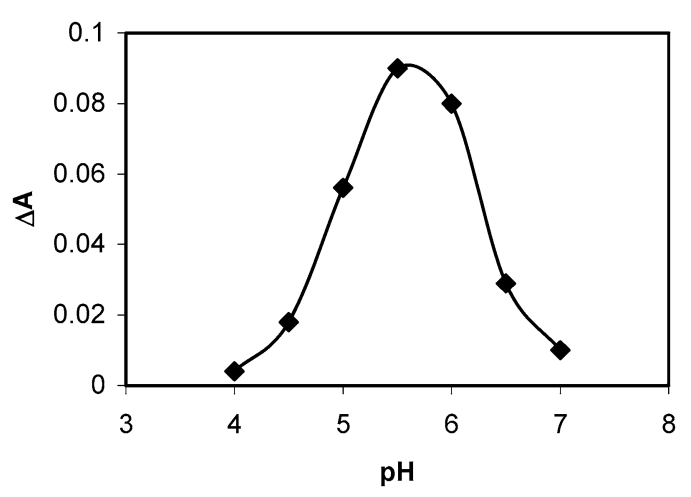

(a)

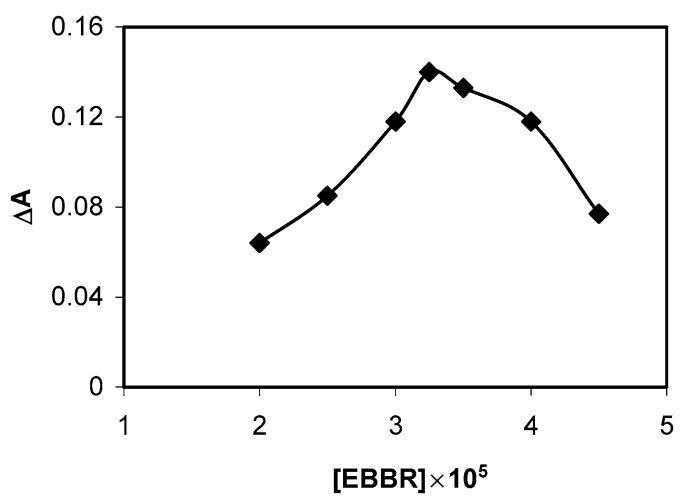

(b)

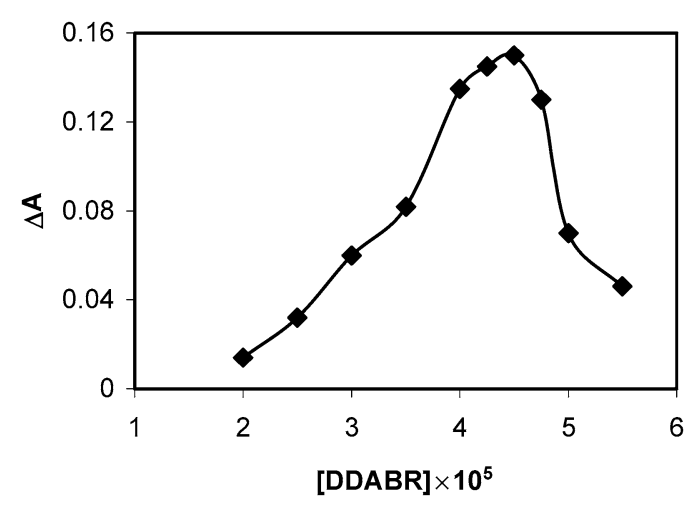

(c)

Fig. 5. Influence of (a) $\mathrm{pH}$, (b) EBBR concentration and (c) DDABr concentration on the measurement parameter $\left(\Delta A=A_{s}-A_{r}\right)$ of EBBR-DDABr-gemfibrozil system. (A) [DDABr] $=4 \times 10^{-5} \mathrm{M}$, [EBBR] $=4 \times 10^{-5} \mathrm{M}$, [gemfibrozil] $=4.0 \mu \mathrm{g} \mathrm{ml}^{-1}$; (B) $\mathrm{pH}=5.5$ and other conditions as $\mathrm{A}$; (B) $\mathrm{pH}=5.5$, [EBBR] $=3.25 \times 10^{-5} \mathrm{M}$ and other conditions as A.

between oppositely charged surfactant and dye molecules [28]. Therefore, acetate buffer disfavored the formation of both dye-surfactant and drug-surfactant aggregates. The effect of buffer on the interaction between dye and surfactant is more pronounced than drug-surfactant interaction, which results in a decrease in the measurement parameter $\left(\Delta A=A_{s}-A_{r}\right)$. A concentration of $0.01 \mathrm{M}$ was chosen as the appropriate amount.

The influence of EBBR concentration was studied in the range of $1 \times 10^{-5}-5 \times 10^{-5} \mathrm{M}$. The results are shown in Fig. 5(b). When the concentration of dye is very lower than the surfactant concentration, the drug molecules will mostly interact with excess surfactant and so there would be no effective competition between the drug and dye molecules. On the other hand, when the dye concentration is much higher than the surfactant concentration, the dye-surfactant interaction is not complete and so $\left[\mathrm{HD}^{2-}\right] /\left[\mathrm{H}_{2} \mathrm{D}^{-}\right]$ ratio and subsequently solution absorbance is not changed considerably by adding drug. According to the results, $3.25 \times 10^{-5} \mathrm{M}$ was selected as the optimum value.

Figure 5(c) shows the effect of DDABr concentration on the measurement parameter. The absorbance difference is reached a maximum at concentration of $4.5 \times 10^{-5} \mathrm{M}$. Therefore, this value was chosen as the optimum concentration for the rest of experiments. Again, at high concentrations of surfactant, the drug is not able to effectively compete with the dye molecules to form aggregates. At lower concentra- 
tions of DDABr, the interaction between the dye and surfactant is not complete and so the absorbance change upon addition of drug is small.

The effect of stirring time was also studied. The absorbance of dye-surfactant and dye-surfactantdrug solutions are slightly decreased by increasing stirring time and remained constant after about five minutes. However, the absorbance difference is remained constant in all cases. We choose the stirring time of $5 \mathrm{~min}$ for obtaining reproducible results.

\subsection{Analytical figures of merit}

In order to obtain the calibration graph, a series of eight standard solutions of gemfibrozil were measured by following the procedure under Section 2. The calibration graph was linear in the range of $0.2-6.0 \mu \mathrm{g} \mathrm{ml}^{-1}$ and its regression equation was:

$$
\Delta A=0.0373( \pm 0.0008) C+0.0022( \pm 0.0029)
$$

where $\Delta A$ is the difference between the absorbance of the EBBR-DDABr system in the presence $\left(A_{s}\right)$ and absence $\left(A_{r}\right)$ of the drug and $C$ is the concentration of gemfibrozil in $\mu \mathrm{g} \mathrm{m}^{-1}$. The values in the parentheses are standard deviations of slope and intercept. The correlation coefficient $(r)$ was 0.998 .

The limits of detection and quantification, calculated as $3 S_{\mathrm{b}} / m$ and $10 S_{\mathrm{b}} / m$ [31] (where $S_{\mathrm{b}}$ is standard deviation of blank and $m$ is slope of calibration graph), were 0.044 and $0.15 \mu \mathrm{g} \mathrm{ml} l^{-1}$, respectively.

In order to study the precision of the proposed method a series of six solutions containing $4.0 \mu \mathrm{g} \mathrm{ml}^{-1}$ gemfibrozil were measured at the same day. By applying the IUPAC definition, the relative error and the relative standard deviation (RSD) were 1.4 and $0.95 \%$, respectively.

\subsection{Application}

Gemfibrozil was satisfactorily determined in the only one pharmaceutical product commercialized in Iran (Gemfibrozil capsules with a nominal content of $300 \mathrm{mg}$ ) by using the proposed method. The obtained result for this sample after five replicate analyses was $296 \pm 9$, which corresponds to $(99 \pm 3) \%$ recovery. Statistical analysis of the assay result showed satisfactory precision of the proposed method with no significant differences between certified and experimental results.

Recovery experiments on pharmaceutical preparations spiked with different amounts of gemfibrozil were also carried out (Table 1). As can be seen, the obtained recoveries are between 97.4 and 104\% and RSDs are between 2 and $4 \%$.

Table 1

Recovery of gemfibrozil added to a pharmaceutical preparation

\begin{tabular}{ccc}
\hline Amount added $(\mu \mathrm{M})^{\mathrm{a}}$ & Amount found $(\mu \mathrm{M})^{\mathrm{b}}$ & Recovery $(\%)$ \\
\hline 4.0 & $3.9 \pm 0.08$ & $97.5 \pm 2.0$ \\
8.0 & $8.3 \pm 0.32$ & $103.8 \pm 3.8$ \\
12.0 & $12.2 \pm 0.29$ & $101.7 \pm 2.4$ \\
16.0 & $16.0 \pm 0.55$ & $100.0 \pm 3.4$ \\
\hline
\end{tabular}

${ }^{\mathrm{a}}$ The given values are concentrations in final solution. ${ }^{\mathrm{b}}$ Average of three determinations $\pm \mathrm{SD}$. 


\section{Conclusion}

A new spectrophotometric methodology based on the competitive aggregation in a dye-surfactantdrug system is developed for the determination of gemfibrozil. It was found that in appropriate chemical conditions the difference between the absorbance of the EBBR-DDABr system in the presence and absence of the drug $\left(A_{s}-A_{r}\right)$ is directly proportional to drug concentration. Therefore, instead of doing photometric titration for each sample to calculate measurement parameter, we simply obtain these absorbance differences. The method was validated and applied to the determination of gemfibrozil in pharmaceutical preparations. This simple and versatile methodology can be used for pharmaceutical quality control of various acid and neutral drugs.

\section{References}

[1] W.L. Hinze, H.N. Singh, Y. Baba and N.G. Harvey, Trends Anal. Chem. 3 (1984), 193-199.

[2] G.L. MacIntire, Crit. Rev. Anal. Chem. 21 (1990), 257-278.

[3] D. Sicilia, S. Rubio and D. Pérez-Bendito, Anal. Chem. 67 (1995), 1872-1880.

[4] E. Borrego, D. Sicilia, S. Rubio and D. Pérez-Bendito, Trends Anal. Chem. 20 (2001), 241-254.

[5] F. Fabios, D. Sicilia, S. Rubio and D. Pérez-Bendito, Anal. Chem. 75 (2003), 6011-6016.

[6] A. Pedraza, M.D. Sicilia, S. Rubio and D. Pérez-Bendito, Anal. Chim. Acta 522 (2004), 89-97.

[7] E.M. Costi, M.D. Sicilia, S. Rubio and D. Pérez-Bendito, Anal. Chim. Acta 549 (2005), 159-165.

[8] A. Pedraza, M.D. Sicilia, S. Rubio and D. Pérez-Bendito, Analyst 131 (2006), 81-89.

[9] C.R. Craig and R.E. Stitzel, Modern Pharmacology, 4th edn, Little, Brown and Company, Boston, 1994, p. 207.

[10] P.A. Tadd and A. Ward, Drugs 36 (1988), 32-35.

[11] E.J. Randinitis, A.W. Kinkel, C. Nelson and T.D. Parker, J. Chromatogr. 307 (1984), 210-215.

[12] A. Hermening, A.K. Gräfe, G. Baktir, E. Mutschler and H. Spahn-Langguth, J. Cromatogr. B 741 (2000), 129-144.

[13] H. Hengy and E.V. Koelle, Arzneim.-Forsch. 35 (1985), 1637-1639.

[14] E.J. Randinitis, T.D. Parker and A.W. Kinkel, J. Chromatogr. 383 (1986), 444-448.

[15] A. Nakagowa, A. Shigeta, H. Iwabuchi, M. Horiguchi, K. Nakamura and H. Takahagi, Biomed. Chromatogr. 5 (1991), $68-73$.

[16] I.B. Kadenatsi, S.N. Levchuk, I.V. Agapitova, M.G. Glezer, V.S. Dombrovskii, M.R. Mukumov and A.A. Firsov, Khim.Farm. Zh. 29 (1995), 61-64.

[17] B.C. Sallustio and B.A. Fairchild, J. Chromatogr. B 665 (1995), 345-353.

[18] E. González-Peñas, S. Agarraberes, A. López-Ocariz, E. García-Quetglas, M.A. Campanero, J.J. Carballal and J. Honorato, J. Pharm. Biomed. Anal. 26 (2001), 7-14.

[19] B.A. Roadcap, D.G. Musson, J. Douglas Rogers and J.J. Zhao, J. Chromatogr. B 791 (2003), 161-170.

[20] M. Farré, I. Ferrer, A. Ginebreda, M. Figueras, L. Olivella, L. Tirapu, M. Vilanova and D. Barceló, J. Chromatogr. 938 (2001), 187-197.

[21] M. Blanco, J. Coello, H. Iturriaga, S. Maspoch and C. de la Pezuela, Appl. Spectrosc. 51 (1997), 240-246.

[22] M. Blanco, J. Coello, H. Iturriaga, S. Maspoch and D. Serrano, Analyst 123 (1998), 2307-2312.

[23] M. Blanco, J. Coello, A. Eustaquio, H. Iturriaga and S. Maspoch, Anal. Chim. Acta 392 (1999), 237-246.

[24] M. Blanco, A. Eustaquio, J.M. Gonzalez and D. Serrano, J. Pharm. Biomed. Anal. 22 (2000), 139-148.

[25] J.L. Manzoori and M. Amjadi, J. Pharm. Biomed. Anal. 31 (2003), 507-513.

[26] B. Tang, B. Jia, G. Cui and Y. Ding, Anal. Chim. Acta 516 (2004), 221-227.

[27] P.D. Tzanavaras and D.G. Themelis, Anal. Lett. 38 (2005), 2165-2173.

[28] M.E. Diaz Garcia and A. Sanz-Medel, Talanta 33 (1986), 255-264.

[29] I. Casero, D. Sicilia, S. Rubio and D. Pérez-Bendito, Talanta 45 (1997), 167-180.

[30] E. Pelizzetti and E. Pramuro, Anal. Chim. Acta 169 (1985), 1-29.

[31] J.D. Winefordner and G.L. Long, Anal. Chem. 55 (1983), 712A-724A. 


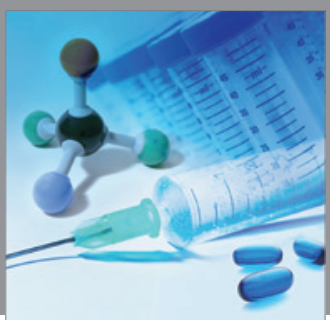

International Journal of

Medicinal Chemistry

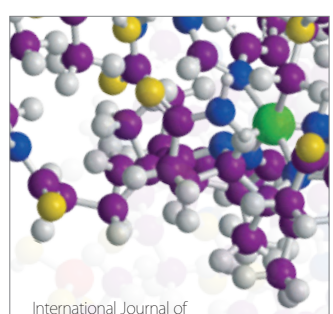

Carbohydrate Chemistry

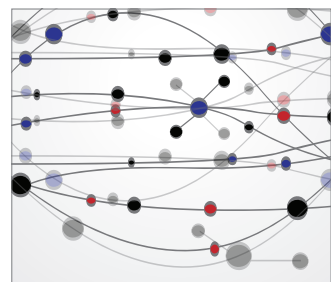

The Scientific World Journal
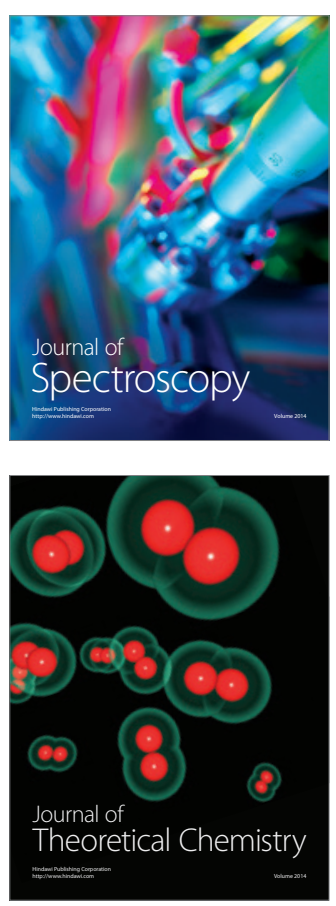
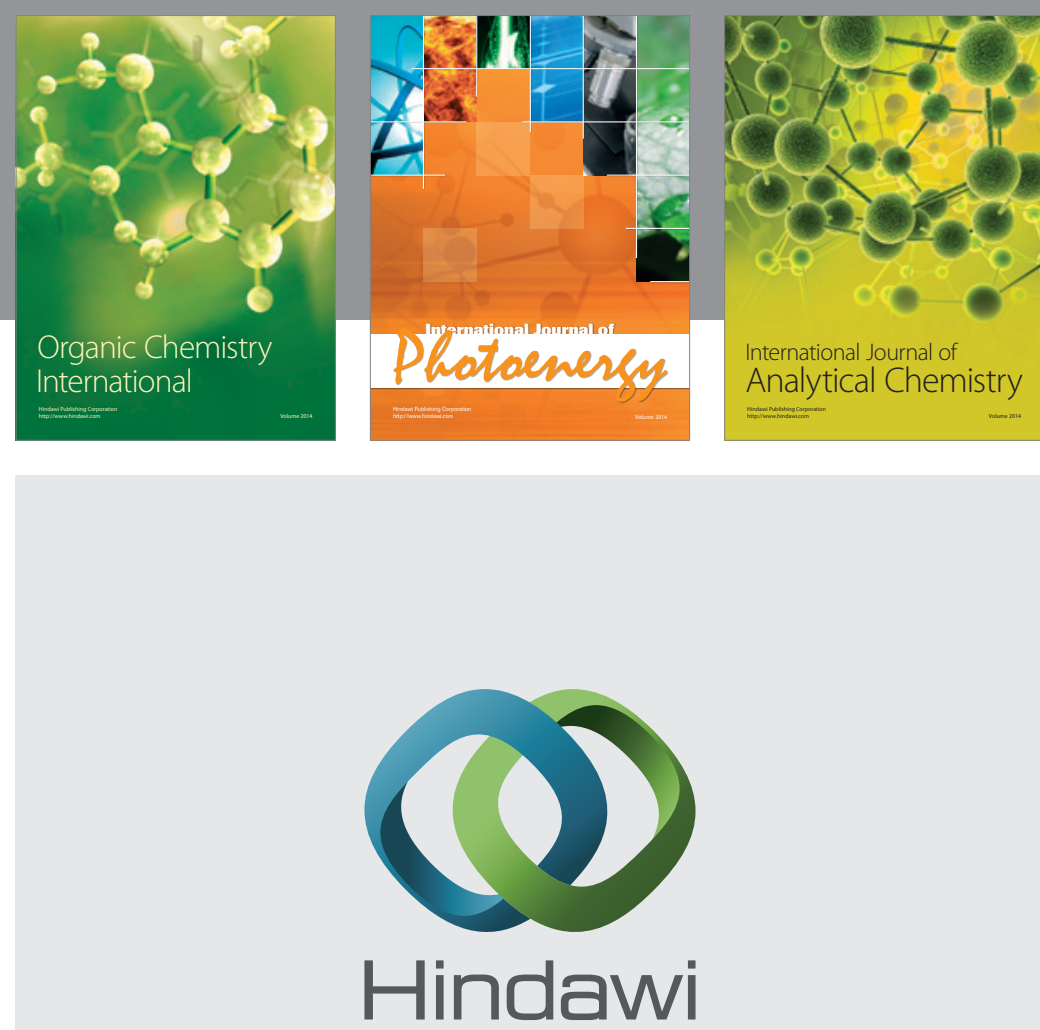

Submit your manuscripts at

http://www.hindawi.com
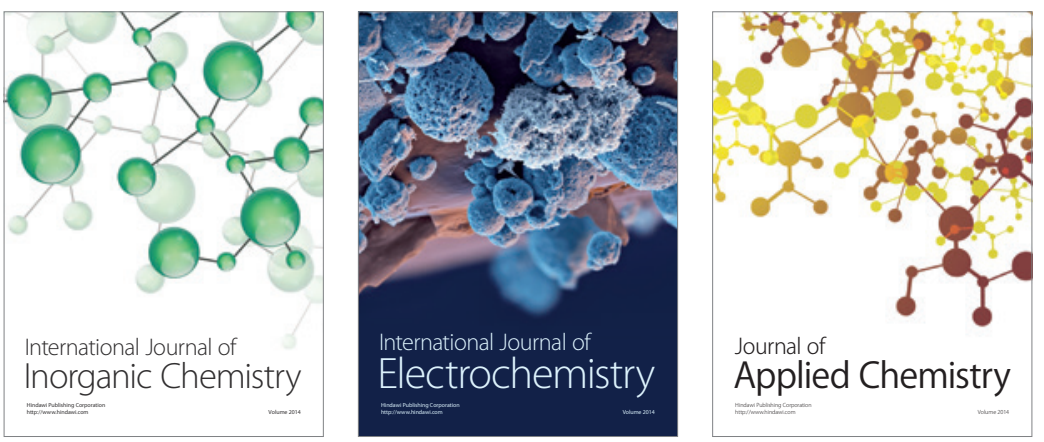

Journal of

Applied Chemistry
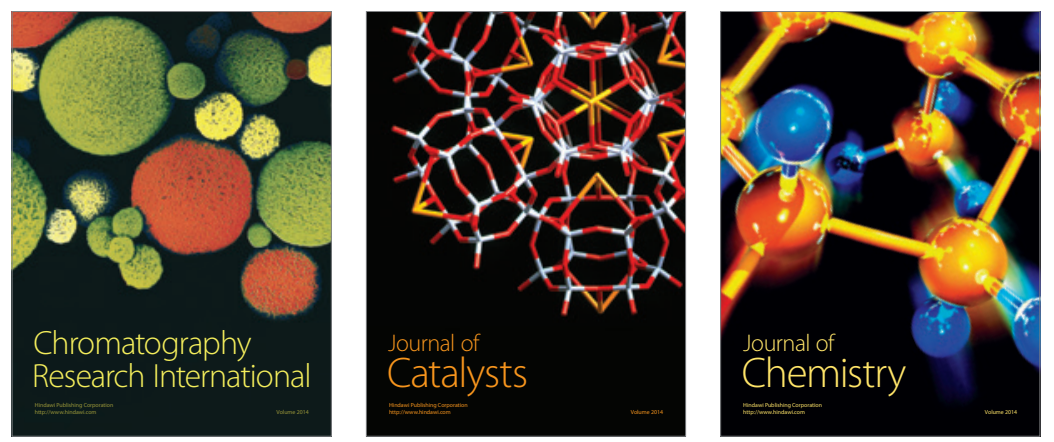
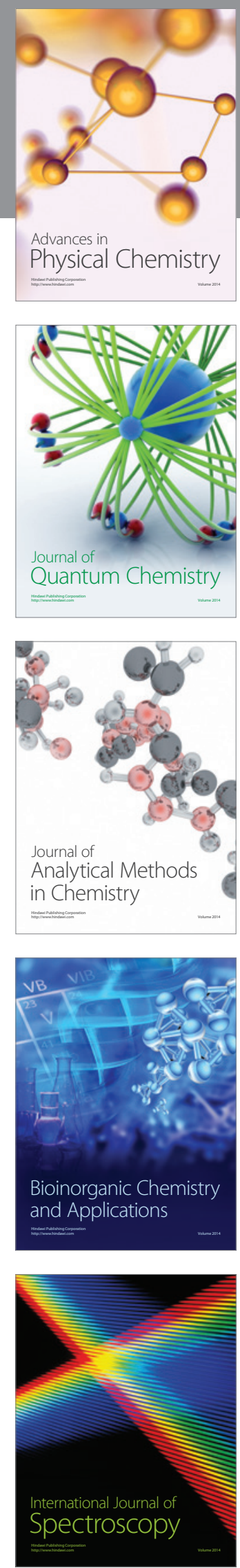